\title{
Quasi-Linear (Stochastic) Partial Differential Equations with Time-Fractional Derivatives *
}

\author{
Wei Liu ${ }^{a \dagger}$, Michael Röckner ${ }^{b}$, José Luís da Silva ${ }^{c}$ \\ a. School of Mathematical Sciences, Jiangsu Normal University, 221116 Xuzhou, China \\ b. Fakultät für Mathematik, Universität Bielefeld, D-33501 Bielefeld, Germany \\ c. CIMA, University of Madeira, Campus da Penteada, 9020-105 Funchal, Portugal
}

\begin{abstract}
In this paper we develop a method to solve (stochastic) evolution equations on Gelfand triples with time-fractional derivative based on monotonicity techniques. Applications include deterministic and stochastic quasi-linear partial differential equations with time-fractional derivatives, including time-fractional (stochastic) porous media equations (including the case where the Laplace operator is also fractional) and $p$ Laplace equations as special cases.
\end{abstract}

AMS Subject Classification: 60H15, 35K59, 45K05, 35K92

Keywords: fractional derivative; monotone; pseudo-monotone; porous media equation; $p$ Laplace equation.

\section{Introduction}

Fractional Calculus has a long history and its origins can be traced back to the end of seventeenth century (cf. [52]). Although the first main steps of the theory date back to the first half of the nineteenth century, this subject became very active only over the last few decades. One of the main reasons is that scientists and engineers have developed many new models that naturally involve fractional differential equations, which have been applied successfully, e.g. in mechanics (theory of viscoelasticity and viscoplasticity)(cf. [37]), bio-chemistry (modelling of polymers and proteins) (cf. [22, 23]), electrical engineering (transmission of ultrasound waves) (cf. [21]), medicine (modelling of human tissue under mechanical loads)(cf. [19, 36]) etc. For more applications and references we refer to [3, 27, 40, 41].

*Financial support by the DFG through the CRC "Taming uncertainty and profiting from randomness and low regularity in analysis, stochastics and their applications" is acknowledged. W.L. is supported by NSFC (No. 11571147), NSF of Jiangsu Province (No. BK20160004), the Qing Lan Project and PAPD of Jiangsu Higher Education Institutions, J.L.S. is supported by project I\&D: UID/MAT/04674/2013.

${ }^{\dagger}$ Corresponding author: weiliu@jsnu.edu.cn 
A typical example of a time-fractional equation is the time-fractional heat equation $\partial_{t}^{\beta} u=$ $\Delta u$ with $0<\beta<1$, where $\partial_{t}^{\beta}$ is the Caputo fractional derivative first appeared in [14] and is defined for $0<\beta<1$ by

$$
\partial_{t}^{\beta} f(t)=\frac{1}{\Gamma(1-\beta)} \frac{d}{d t} \int_{0}^{t}(t-s)^{-\beta}(f(s)-f(0)) d s,
$$

where $\Gamma$ is the Gamma function defined by $\Gamma(\lambda):=\int_{0}^{\infty} t^{\lambda-1} e^{-t} d t$. Here one implicitly assumes that $f$ is such that the integral on the right-hand side is (weakly) differentiable in $t$. For the precise domain of $f$ for which $\partial_{t}^{\beta} f$ is defined and which is convenient in our case, we refer to (2.11) below.

The classical heat equation $\partial_{t} u=\Delta u$ describes heat propagation in homogeneous medium. The time-fractional diffusion equation $\partial_{t}^{\beta} u=\Delta u$ with $0<\beta<1$ has been widely used to model anomalous diffusions exhibiting subdiffusive behavior, e.g. due to particle sticking and trapping phenomena (cf. [40, 54]). While in normal diffusions (described by the heat equation or more general parabolic equations), the mean squared displacement of a diffusive particle behaves like const. $t$ for $t \rightarrow \infty$, the time-fractional diffusion equation exhibits a behaviour like const $t^{\beta}$ for $t \rightarrow \infty$. This is the reason why time-fractional equations with $0<\beta<1$ are called subdiffusion equations in the literature, and for the case $1<\beta<2$ are called superdiffusion equations. In fact, there is a diverse number of real world systems which demonstrate this type of phenomena. For example, the above time-fractional equations and nonlinear variants of them are also widely used to model dynamical processes in materials with memory, e.g. the diffusion of fluids in porous media with memory (see [13]). We refer to the survey article [42] and the monographs [3, 45, 48, 49, 53, 67] for more references.

We also want to mention the interesting interplay between stochastic processes and timefractional differential equations. A celebrated result of Einstein established a mathematical link between random walks, the diffusion equation and Brownian motion. The probability densities of the Brownian motion solve a diffusion equation (heat equation), and hence we refer to the Brownian motion as the stochastic solution to the heat equation. Similarly, the stochastic solutions of time-fractional diffusion equations are subordinated processes (e.g. iterated Brownian motion). The solutions to fractional diffusion equations are strictly related to stable densities. Indeed, the stochastic solutions of time-fractional diffusions can be realized through "time-change" by inverse stable subordinators. Just as Brownian motion is a scaling limit of simple random walks, the stochastic solutions to certain fractional diffusion equations are scaling limits of continuous time random walks, in which the i.i.d. jumps are separated by i.i.d. waiting times. For this subject we refer to [2, 39, 46] and the references therein.

In recent years, there has been also growing interest in stochastic time-fractional partial differential equations (see e.g. [20, 56]). For example, the author in [66] considers stochastic Volterra equations with singular kernels in a 2-smooth Banach space. The authors in [18] study the $L^{2}$-theory for a class of semilinear SPDEs with time-fractional derivatives, which can be used to describe random effects on transport of particles in media with thermal memory, or particles subject to sticking and trapping. In [43, 44, the authors consider a space-time fractional stochastic heat type equation to model phenomenon with random 
effects with thermal memory, and they prove existence and uniqueness of mild solutions and also some intermittency property (see also [24]). For a linear stochastic partial differential equation of fractional order both in the time and space variables with a different type of noise term we refer to [16, 26]. In [15, 17] the authors investigate linear stochastic time-fractional partial differential equations for the more general case $0<\beta \leq 2$.

In this paper, we are mainly interested in non-linear stochastic time-fractional partial differential equations in a variational setting, which include in particular important examples of quasi-linear type as the stochastic porous media or the $p$-Laplace equation. They are of the following form:

$$
\partial_{t}^{\beta} X(t)+A(t, X(t))=\partial_{t}^{\gamma} \int_{0}^{t} B(s) d W(s), 0<t<T,
$$

where $V \subseteq H \equiv H^{*} \subseteq V^{*}$ is a Gelfand triple, $\beta \in(0,1], \gamma \in\left(0, \beta+\frac{1}{2}\right) \cap(0,1], \partial_{t}^{\beta}$ is the Caputo fractional derivative, $A:[0, T] \times V \rightarrow V^{*}$ and $B:[0, T] \rightarrow L_{H S}(U ; H)$ (here $L_{H S}(U ; H)$ denotes the space of all Hilbert-Schmidt operators from $U$ to $\left.H\right)$ are measurable. For simplicity we only consider the additive type noise in (1.2) here, the case of general multiplicative noise will be investigated in a forthcoming paper.

We will establish the existence and uniqueness of solutions to (1.2) under classical monotonicity and coercivity conditions on $A$ (see Theorems 2.1 and 2.7). The proof of the main results will be based on a functional analytic approach, which, to the best of our knowledge, is new to solve both the deterministic and stochastic time-fractional partial differential equations in the variational setting. This approach is inspired by the work of Stannat [57] on the theory of generalized Dirichlet forms, which in turn draws essential ideas from [30]. We will establish a general existence result concerning an abstract operator equation (see Theorem 2.3 below) which is central to our approach.

We should mention that time-fractional linear evolution equations in the Gelfand triple setting have first been investigated in [62]. Later on the author also proved the global solvability of a non-degenerate parabolic equation with time-fractional derivative in 63] (cf. [1] for more general case). However, these results cannot be applied to quasilinear type equations like the porous media or the $p$-Laplace equation. In [28 the authors investigate elliptic-parabolic integro-differential equations with $L^{1}$-data. Their framework includes the time-fractional $p$-Laplace equation. However, the authors in [28] consider generalized solutions (i.e. entropy solutions). Therefore, the results of this paper generalize or complement the corresponding results in [28, 62, 63] in the setting of time-fractional quasilinear PDE with monotone coefficients. Recently, the authors in [61] derive very interesting decay estimates for the solutions of time-fractional porous medium and $p$-Laplace equations (by assuming the existence), and the decay behaviour is notably different from the case with usual time derivative. Hence, here we, in particular, give a positive answer to the question of the existence and uniqueness of solutions to the time-fractional porous medium equations and $p$-Laplace equations, which are left open in [61].

If $\beta=\gamma=1$, then both the deterministic and stochastic equation (1.2) have been intensively investigated in the variational setting. For the deterministic case, one might refer to the monographs $4,6,30,55,64$ and the references therein. But also the stochastic 
case (SPDE) has attracted more and more attention in recent years, we refer to some classical references [7, 29, 47] and recent works [6, 12, 25, 32, 33, 34, 35, 51, 65] (see also the references therein).

The paper is organized as follows. In Section 2 we present the main results on the existence and uniqueness of solutions to deterministic and stochastic nonlinear evolution equations with time-fractional derivatives. In Section 3 we will apply our main results to stochastic time-fractional porous medium equations and $p$-Laplace equations as model examples. The well-posedness of both equations have been open problems even in the deterministic case.

\section{Main Results}

Let $\left(H,\langle\cdot, \cdot\rangle_{H}\right)$ be a real separable Hilbert space identified with its dual space $H^{*}$ by the Riesz isomorphism. Let $V$ be a real reflexive Banach space, continuously and densely embedded into $H$. Then we have the following Gelfand triple

$$
V \subseteq H \equiv H^{*} \subseteq V^{*}
$$

Let $V^{*}\langle\cdot, \cdot\rangle_{V}$ denote the dualization between $V$ and its dual space $V^{*}$. Then it is easy to show that

$$
V^{*}\langle u, v\rangle_{V}=\langle u, v\rangle_{H}, \quad u \in H, v \in V .
$$

Now, for $T \in[0, \infty)$ fixed, we consider the following general nonlinear evolution equation with time-fractional derivative

$$
\partial_{t}^{\beta}(u(t)-x)+A(t, u(t))=f(t), 0<t<T,
$$

where

$$
\partial_{t}^{\beta}(u-x):=\frac{1}{\Gamma(1-\beta)} \frac{d}{d t} \int_{0}^{t}(t-s)^{-\beta}(u(s)-x) d s,
$$

is the Riemann-Liouville fractional derivative, which coincides with the Caputo fractional derivative if $u(0)=x$ (i.e. considered $x$ as the initial condition), $A:[0, \infty) \times V \rightarrow V^{*}$ is restrictedly measurable, i.e. for each $d t$-version of $u \in L^{1}([0, \infty) ; V), t \mapsto A(t, u(t))$ is $V^{*}$-measurable on $[0, \infty)$, and $f \in L^{1}\left([0, \infty) ; V^{*}\right)$.

Applying the Riemann-Liouville fractional integral $I_{t}^{\beta}$, defined by

$$
I_{t}^{\beta} f(t):=\frac{1}{\Gamma(\beta)} \int_{0}^{t}(t-s)^{\beta-1} f(s) d s=:\left(g_{\beta} * f\right)(t),
$$

to equation (2.1) one obtains that

$$
u(t)=x-\frac{1}{\Gamma(\beta)} \int_{0}^{t}(t-s)^{\beta-1} A(s, u(s)) d s+\frac{1}{\Gamma(\beta)} \int_{0}^{t}(t-s)^{\beta-1} f(s) d s,
$$

for $d t$-a.e. $t \in[0, \infty)$. 
Here we recall that $\frac{d}{d t}$ in the definition of $\partial_{t}^{\beta}$ (see (1.1) ) is understood as a weak derivative. Defining $\tilde{u}$ to be equal to the right-hand side of (2.2), then this $d t$-version of $u$ satisfies $\tilde{u}(0)=x$. Concerning the continuity properties of $\tilde{u}$ we refer to Theorem 2.1 below. We also remark that below we work with functions $u$ only determined $d t$-a.e., so $u(0)$ can be chosen arbitrarily. Therefore, we write $x$ in (2.1) instead of $u(0)$.

Now let us formulate the precise conditions on the coefficients in (2.1). Suppose for fixed $\alpha>1$ that there exist constants $\delta>0, C$ and $g \in L^{1}\left([0, \infty) ; \mathbb{R}_{+}\right)$such that the following conditions hold for all $t \in[0, \infty)$ and $v, v_{1}, v_{2} \in V$.

(H1) (Hemicontinuity) The map $s \mapsto V^{*}\left\langle A\left(t, v_{1}+s v_{2}\right), v\right\rangle_{V}$ is continuous on $\mathbb{R}$.

(H2) (Monotonicity)

$$
V^{*}\left\langle A\left(t, v_{1}\right)-A\left(t, v_{2}\right), v_{1}-v_{2}\right\rangle_{V} \geq 0
$$

(H3) (Coercivity)

$$
V^{*}\langle A(t, v), v\rangle_{V} \geq \delta\|v\|_{V}^{\alpha}-g(t)
$$

(H4) (Growth)

$$
\|A(t, v)\|_{V^{*}} \leq g(t)^{\frac{\alpha-1}{\alpha}}+C\|v\|_{V}^{\alpha-1}
$$

We define the following spaces,

$$
\begin{aligned}
\mathcal{V} & =L^{\alpha}([0, \infty) ; V) \cap L^{2}([0, \infty) ; H), \\
\mathcal{H} & =L^{2}([0, \infty) ; H), \\
\mathcal{V}^{*} & =L^{\frac{\alpha}{\alpha-1}}\left([0, \infty) ; V^{*}\right)+L^{2}([0, \infty) ; H),
\end{aligned}
$$

where $\|\cdot\|_{\mathcal{V}}:=\max \left(\|\cdot\|_{L^{\alpha}([0, \infty) ; V)},\|\cdot\|_{\mathcal{H}}\right)$ and for $u \in \mathcal{V}^{*}$

$\|u\|_{\mathcal{V}^{*}}:=\inf \left\{\left\|u_{1}\right\|_{L^{\frac{\alpha}{\alpha-1}}\left([0, \infty) ; V^{*}\right)}+\left\|u_{2}\right\|_{\mathcal{H}}: u_{1} \in L^{\frac{\alpha}{\alpha-1}}\left([0, \infty) ; V^{*}\right), u_{2} \in \mathcal{H}\right.$ such that $\left.u=u_{1}+u_{2}\right\}$.

Then for $x=0$ (the case for general initial condition $x$ will then follow easily as we shall see below) the original equation (2.1) can be rewritten in the following form

$$
\partial_{t}^{\beta} u+\mathcal{A} u=f
$$

where

$$
\mathcal{A}: \mathcal{V} \rightarrow \mathcal{V}^{*} ;(\mathcal{A} u)(t)=A(t, u(t)), t \in[0, \infty)
$$

It is easy to see that $\mathcal{A}: \mathcal{V} \rightarrow \mathcal{V}^{*}$ is monotone, coercive and bounded on bounded sets.

We shall see below that $\partial_{t}^{\beta}$ with domain $\left\{\left.u \in \mathcal{H}|r \mapsto| r\right|^{\beta} \hat{u}(r) \in L^{2}(\mathbb{R} ; H)\right\} \cap \mathcal{V}$ is closable as an operator from $\mathcal{V}$ to $\mathcal{V}^{*}$. Let $\left(\partial_{t}^{\beta}, \mathcal{F}\right)$ denote its closure with norm $\|u\|_{\mathcal{F}}:=$ $\left(\|u\|_{\mathcal{V}}^{2}+\left\|\partial_{t}^{\beta} u\right\|_{\mathcal{V}^{*}}^{2}\right)^{\frac{1}{2}}, u \in \mathcal{F}$. Here $\hat{u}$ denotes the Fourier transform of $u$, considered as a function from $\mathbb{R}$ to $H$, setting $u \equiv 0$ on $\mathbb{R} \backslash(-\infty, 0)$. 
Theorem 2.1. Suppose that $T \in[0, \infty)$ and $A:[0, \infty) \times V \rightarrow V^{*}$ satisfies $(H 1)$ - $(H 4)$. Then for every $x \in V$ and $f \in \mathcal{V}^{*}$, (2.1) has a unique solution $u$ such that $u-x \varphi \in \mathcal{F}$ for every $\varphi \in L^{\alpha}([0, \infty) ; \mathbb{R})$ with $\varphi \equiv 1$ on $[0, T+1)$. In particular,

$$
u-x \varphi \in L^{\alpha}([0, \infty) ; V) ; \partial_{t}^{\beta}(u-x \varphi) \in L^{\frac{\alpha}{\alpha-1}}\left([0, \infty) ; V^{*}\right)
$$

and for $d t$-a.e. $t \in[0, T]$,

$$
u(t)=x-\frac{1}{\Gamma(\beta)} \int_{0}^{t}(t-s)^{\beta-1} A(s, u(s)) d s+\frac{1}{\Gamma(\beta)} \int_{0}^{t}(t-s)^{\beta-1} f(s) d s .
$$

Furthermore, $t \mapsto \frac{1}{\Gamma(1-\beta)} \int_{0}^{t}(t-s)^{-\beta}(u(s)-x \varphi(s)) d s$ has a continuous $H$-valued $d t$-version, and if $\beta \in\left(\frac{\alpha-1}{\alpha}, 1\right), t \mapsto u(t)$ has a continuous $V^{*}$-valued $d t$-version.

Remark 2.1. ( $i$ ) As a matter of fact, the monotonicity assumption (H2) of $A$ is a sufficient condition to imply that $\mathcal{A}: \mathcal{V} \rightarrow \mathcal{V}^{*}$ is pseudo-monotone. The above theorem still holds if we replace (H2) by assuming $\mathcal{A}: \mathcal{V} \rightarrow \mathcal{V}^{*}$ is pseudo-monotone. In [34, 35, a local monotonicity condition is assumed for $A$ which yields that $A(t, \cdot): V \rightarrow V^{*}$ is pseudo-monotone provided $V \subseteq H$ is a compact embedding. However, it is still not clear whether it implies that $\mathcal{A}: \mathcal{V} \rightarrow \mathcal{V}^{*}$ is pseudo-monotone.

(ii) It is easy to see from the proof that to have uniqueness of solutions one can replace (H2) by the following weak monotonicity:

$$
V^{*}\left\langle A\left(t, v_{1}\right)-A\left(t, v_{2}\right), v_{1}-v_{2}\right\rangle_{V}+K\left\|v_{1}-v_{2}\right\|_{H}^{2} \geq 0,
$$

where $K$ is a positive constant.

(iii) If $A$ is the subdifferential of a convex function, i.e.

$$
A(t, u)=\partial \varphi(t, u), u \in V
$$

where $\varphi(t, \cdot): V \rightarrow \mathbb{R}$ is convex and continuous, then for

$$
\Phi(u):=\int_{0}^{T} \varphi(t, u(t)) d t, u \in \mathcal{V}
$$

we have $\partial \Phi(u)=\mathcal{A}(u), \forall u \in \mathcal{V}$. Then

$$
\begin{aligned}
& \mathcal{A}(u)-\Lambda u=f \\
\Leftrightarrow & \partial \Phi(u)-\Lambda u=f \\
\Leftrightarrow & u=\arg \min _{v \in \mathcal{V}}\left\{\Phi(v)+\Phi^{*}(f+\Lambda v)-\mathcal{V}^{*}\langle\Lambda v, v\rangle_{\mathcal{V}}-\mathcal{V}^{*}\langle f, v\rangle_{\mathcal{V}}\right\},
\end{aligned}
$$

where $\Phi^{*}(\eta):=\sup _{v \in \mathcal{V}}\left\{\mathcal{V}^{*}\langle\eta, v\rangle_{\mathcal{V}}-\Phi(v)\right\}$. 
Now we recall the definition of pseudo-monotone operator, which is a very useful generalization of monotone operator and was first introduced by Brézis in [8]. We use the notation "-" for weak convergence in Banach spaces.

Definition 2.1. The operator $M: \mathcal{V} \rightarrow \mathcal{V}^{*}$ is called pseudo-monotone if $v_{n} \rightarrow v$ in $\mathcal{V}$ as $n \rightarrow \infty$ and

$$
\limsup _{n \rightarrow \infty} \mathcal{V}^{*}\left\langle M\left(v_{n}\right), v_{n}-v\right\rangle_{\mathcal{V}} \leq 0
$$

implies for all $u \in \mathcal{V}$

$$
\mathcal{V}^{*}\langle M(v), v-u\rangle_{\mathcal{V}} \leq \liminf _{n \rightarrow \infty} \mathcal{V}^{*}\left\langle M\left(v_{n}\right), v_{n}-u\right\rangle_{\mathcal{V}}
$$

Remark 2.2. Browder introduced a slightly different definition of a pseudo-monotone operator in [11]: An operator $M: \mathcal{V} \rightarrow \mathcal{V}^{*}$ is called pseudo-monotone if $v_{n} \rightarrow v$ in $\mathcal{V}$ as $n \rightarrow \infty$ and

$$
\limsup _{n \rightarrow \infty}\left\langle M\left(v_{n}\right), v_{n}-v\right\rangle_{\mathcal{V}} \leq 0
$$

implies

$$
M\left(v_{n}\right) \rightarrow M(v) \text { and } \lim _{n \rightarrow \infty} \mathcal{V}^{*}\left\langle M\left(v_{n}\right), v_{n}\right\rangle_{\mathcal{V}}=\mathcal{V}^{*}\langle M(v), v\rangle_{\mathcal{V}}
$$

In particular, if $M$ is bounded on bounded sets, then these two definitions are equivalent, we refer to [34, 35].

Lemma 2.2. If $M: \mathcal{V} \rightarrow \mathcal{V}^{*}$ is pseudo-monotone, bounded on bounded sets and coercive, then $M$ is surjective, i.e. for any $f \in \mathcal{V}^{*}$, the equation $M u=f$ has a solution.

Proof. This is a classical result due to Brézis. For the proof we refer to [8] or [64, Theorem 27.A].

As in [57] we consider a generator $\Lambda$, with domain $D(\Lambda, \mathcal{H})$, of a $C_{0}$-contraction semigroup of linear operators on $\mathcal{H}$ whose restrictions to $\mathcal{V}$ form a $C_{0}$-semigroup of linear operators on $\mathcal{V}$. The generator of the latter is again $\Lambda$, but with domain $D(\Lambda, \mathcal{V}):=\{u \in \mathcal{V} \cap D(\Lambda, \mathcal{H}) \mid$ $\Lambda u \in \mathcal{V}\}$. Then $D(\Lambda, \mathcal{V})$ is dense in $\mathcal{V}$, hence so is $D(\Lambda, \mathcal{H}) \cap \mathcal{V}$.

By [57, Lemma 2.3], $\Lambda: D(\Lambda, \mathcal{H}) \cap \mathcal{V} \rightarrow \mathcal{V}^{*}$ is closable as an operator from $\mathcal{V}$ to $\mathcal{V}^{*}$. Denoting its closure by $(\Lambda, \mathcal{F}), \mathcal{F}$ is a Banach space with norm $\|u\|_{\mathcal{F}}:=\left(\|u\|_{\mathcal{V}}^{2}+\|\Lambda u\|_{\mathcal{V}^{*}}^{2}\right)^{\frac{1}{2}}$, $u \in \mathcal{F}$.

We will use the following abstract result in which $\Lambda$ will later be taken to be $-\partial_{t}^{\beta}$ to solve equation (2.1). This abstract result is a generalization of [57, Proposition 3.2]. We replace the strong monotonicity assumption in [57, Proposition 3.2] by the classical monotonicity $(H 2)$ (see also Remark 2.1) and consider a reflexive Banach space $\mathcal{V}$ in place of the Hilbert space $\mathcal{V}$ in [57].

Theorem 2.3. For any $f \in \mathcal{V}^{*}$, there exists a solution $u \in \mathcal{F}$ of the equation $\mathcal{A} u-\Lambda u=f$. 
Proof. Step 1: For $\alpha>0$, consider the Yosida approximation $\Lambda_{\alpha}: \mathcal{V} \rightarrow \mathcal{V}^{*}$ defined by

$$
\Lambda_{\alpha}=\alpha\left(\alpha V_{\alpha}-I\right)
$$

where $V_{\alpha}=(\alpha-\Lambda)^{-1}, \alpha>0$, is the resolvent of $(\Lambda, D(\Lambda, \mathcal{H}))($ on $\mathcal{H})$.

Note that $\mathcal{A}-\Lambda_{\alpha}$ is pseudo-monotone, coercive and bounded on bounded sets. By Lemma 2.2 there exists $u_{\alpha} \in \mathcal{V}$ such that $\mathcal{A} u_{\alpha}-\Lambda_{\alpha} u_{\alpha}=f$.

Step 2: Note that

$$
\mathcal{V}^{*}\left\langle\mathcal{A} u_{\alpha}, u_{\alpha}\right\rangle_{\mathcal{V}} \leq \mathcal{V}^{*}\left\langle\mathcal{A} u_{\alpha}-\Lambda_{\alpha} u_{\alpha}, u_{\alpha}\right\rangle_{\mathcal{V}}=\mathcal{V}^{*}\left\langle f, u_{\alpha}\right\rangle_{\mathcal{V}} \leq\|f\|_{\mathcal{V}^{*}}\left\|u_{\alpha}\right\|_{\mathcal{V}}
$$

Hence, by the coercivity assumption (H3) we obtain that $\sup _{\alpha>0}\left\|u_{\alpha}\right\|_{\mathcal{V}}<\infty$, and hence $\sup _{\alpha>0}\left\|\mathcal{A} u_{\alpha}\right\|_{\mathcal{V}^{*}}<\infty$ by $(H 4)$.

Since for any $v \in \mathcal{V}$

$$
\begin{aligned}
\mathcal{V}^{*}\left\langle\Lambda_{\alpha} u_{\alpha}, v\right\rangle_{\mathcal{V}} & =-\mathcal{V}^{*}\left\langle\mathcal{A} u_{\alpha}-\Lambda_{\alpha} u_{\alpha}, v\right\rangle_{\mathcal{V}}+\mathcal{V}^{*}\left\langle\mathcal{A} u_{\alpha}, v\right\rangle_{\mathcal{V}} \\
& =-\mathcal{V}^{*}\langle f, v\rangle_{\mathcal{V}}+\mathcal{V}^{*}\left\langle\mathcal{A} u_{\alpha}, v\right\rangle_{\mathcal{V}} \\
& \leq\left(\|f\|_{\mathcal{V}^{*}}+\left\|\mathcal{A} u_{\alpha}\right\|_{\mathcal{V}^{*}}\right)\|v\|_{\mathcal{V}}
\end{aligned}
$$

we have $\sup _{\alpha>0}\left\|\Lambda_{\alpha} u_{\alpha}\right\|_{\mathcal{V}^{*}}<\infty$.

By the apriori estimates above we know there exists a subsequence $\alpha_{n} \rightarrow \infty$ such that

$$
\begin{aligned}
u_{\alpha_{n}} \rightarrow u & \text { in } \mathcal{V} ; \\
\mathcal{A} u_{\alpha_{n}} \rightarrow h & \text { in } \mathcal{V}^{*} ; \\
\Lambda_{\alpha_{n}} u_{\alpha_{n}} \rightarrow g & \text { in } \mathcal{V}^{*} .
\end{aligned}
$$

So, it is easy to see that $h-g=f$.

Note that

$$
\alpha_{n} V_{\alpha_{n}} u_{\alpha_{n}} \rightarrow u \text { in } \mathcal{V}
$$

So we have

$$
\Lambda \alpha_{n} V_{\alpha_{n}} u_{\alpha_{n}} \rightarrow g \text { in } \mathcal{V}^{*}
$$

since $\Lambda \alpha_{n} V_{\alpha_{n}} u_{\alpha_{n}}=\Lambda_{\alpha_{n}} u_{\alpha_{n}}$. Hence $\Lambda u=g$. Note that $\left\|\alpha_{n} V_{\alpha_{n}} u_{\alpha_{n}}\right\|_{\mathcal{V}} \leq 2 C\left\|u_{\alpha_{n}}\right\|_{\mathcal{V}}$, hence we have

$$
\sup _{n}\left\|\alpha_{n} V_{\alpha_{n}} u_{\alpha_{n}}\right\|_{\mathcal{F}}<\infty
$$

which implies that $u \in \mathcal{F}$.

Step 3: Now we only need to show $\mathcal{A} u=h$.

Since $u_{\alpha_{n}} \rightarrow u$

$$
\begin{aligned}
& \limsup _{n \rightarrow \infty} \mathcal{V}^{*}\left\langle\mathcal{A} u_{\alpha_{n}}, u_{\alpha_{n}}-u\right\rangle_{\mathcal{V}} \\
= & \limsup _{n \rightarrow \infty}\left\langle\Lambda_{\alpha_{n}} u_{\alpha_{n}}+f, u_{\alpha_{n}}-u\right\rangle_{\mathcal{V}} \\
= & \limsup _{n \rightarrow \infty}\left\langle\Lambda_{\alpha_{n}} u_{\alpha_{n}}, u_{\alpha_{n}}-u\right\rangle_{\mathcal{V}} \\
\leq & \mathcal{V}^{*}\langle\Lambda u, u-u\rangle_{\mathcal{V}}=0,
\end{aligned}
$$


where the inequality follows from $\mathcal{V}^{*}\left\langle\Lambda_{\alpha_{n}}\left(u_{\alpha_{n}}-u\right), u_{\alpha_{n}}-u\right\rangle_{\mathcal{V}} \leq 0$.

So, we have

$$
\limsup _{n \rightarrow \infty} \mathcal{V}^{*}\left\langle\mathcal{A} u_{\alpha_{n}}, u_{\alpha_{n}}\right\rangle_{\mathcal{V}} \leq \mathcal{V}^{*}\langle h, u\rangle_{\mathcal{V}}
$$

Hence, by the pseudomonotonicity, we have for any $w \in \mathcal{V}$

$$
\begin{aligned}
& \mathcal{V}^{*}\langle\mathcal{A} u, u-w\rangle_{\mathcal{V}} \\
\leq & \liminf _{n \rightarrow \infty} \mathcal{V}^{*}\left\langle\mathcal{A} u_{\alpha_{n}}, u_{\alpha_{n}}-w\right\rangle_{\mathcal{V}} \\
\leq & \liminf _{n \rightarrow \infty} \mathcal{V}^{*}\left\langle\mathcal{A} u_{\alpha_{n}}, u_{\alpha_{n}}\right\rangle_{\mathcal{V}}-\mathcal{V}^{*}\langle h, w\rangle_{\mathcal{V}} \\
\leq & \mathcal{V}^{*}\langle h, u-w\rangle_{\mathcal{V}},
\end{aligned}
$$

which implies $\mathcal{A} u=h$ since $w \in \mathcal{V}$ was arbitrary.

Now we want to show that we can take $\Lambda:=-\partial_{t}^{\beta}$, i.e., we have to show that $-\partial_{t}^{\beta}$ generates a $C_{0}$-semigroup of contractions on $\mathcal{H}$ which can be restricted to a $C_{0}$-semigroup on $\mathcal{V}$. To this end, let us define the following "shift to the right" semigroup $U_{t}, t>0$, on $\mathcal{H}$. For $f \in \mathcal{H}, t \geq 0$, define

$$
U_{t} f(r):=\mathbb{1}_{[0, \infty)}(r-t) f(r-t), r \in[0, \infty)
$$

Then it is trivial to check that $\left(U_{t}\right)_{t>0}$ is a $C_{0}$-contraction semigroup on $\mathcal{H}$ and it obviously can be restricted to a $C_{0}$-semigroup on $\mathcal{V}$ (even in this case consisting also of contractions on $\mathcal{V})$. Now fix $\beta \in(0,1)$ and define for $f \in \mathcal{H}$

$$
U_{t}^{\beta} f:=\int_{0}^{\infty} U_{s} f \eta_{t}^{\beta}(d s), t \geq 0
$$

where $\left(\eta_{t}^{\beta}\right)_{t>0}$ denotes the one-sided stable semigroup (of probability measures on $([0, \infty), \mathcal{B}([0, \infty)))$ ) of order $\beta$, i.e. we have for its Fourier transform

$$
\hat{\eta}_{t}^{\beta}(s)=\int_{0}^{\infty} e^{i s r} \eta_{t}^{\beta}(d r)=e^{-t(i s)^{\beta}}, s \in \mathbb{R}, t>0 .
$$

We note here that

$$
(i s)^{\beta}=\left[\cos \left(\frac{\beta \pi}{2} \operatorname{sign}(s)\right)+i \sin \left(\frac{\beta \pi}{2} \operatorname{sign}(s)\right)\right]|s|^{\beta}, s \in \mathbb{R},
$$

hence $\eta_{t}^{\beta}(d s)$ is absolutely continuous with respect to Lebesgue measure $d s$. It is a well-known fact (see e.g. [38, Chap. II, Sect. 4b]), that $\left(U_{t}^{\beta}\right)_{t>0}$ is also a $C_{0}$-semigroup of contractions on $\mathcal{H}$ which obviously can be restricted to a $C_{0}$-semigroup on $\mathcal{V}$ (again consisting of contractions). 
Proposition 2.4. The generator $(\Lambda, D(\Lambda, \mathcal{H}))$ of $\left(U_{t}^{\beta}\right)_{t>0}$ (on $\left.\mathcal{H}\right)$ is given as follows

$$
\begin{gathered}
D(\Lambda, \mathcal{H})=\left\{\left.u \in \mathcal{H}|r \mapsto| r\right|^{\beta} \hat{u}(r) \in L^{2}(\mathbb{R} ; H)\right\}, \\
(\Lambda u)^{\wedge}(r)=-(i r)^{\beta} \hat{u}(r), r \in \mathbb{R},
\end{gathered}
$$

where $\hat{u}$ denotes the Fourier transform of $u$ and is considered as a function from $\mathbb{R}$ to $H$. In particular, if $\beta>\frac{1}{2}$, then each $u \in D(\Lambda, \mathcal{H})$ has a dt-version $\tilde{u}$ such that $[0, \infty) \ni t \mapsto$ $\tilde{u}(t) \in H$ is continuous and $\tilde{u}(0)=0$.

Proof. Below we consider each $\eta_{t}^{\beta}(d s)$ as a measure on all of $\mathbb{R}$, by defining

$$
\eta_{t}^{\beta}(A):=\eta_{t}^{\beta}(A \cap[0, \infty)), A \in \mathcal{B}(\mathbb{R}),
$$

and any function $f$ in $\mathcal{H}$ as a function on $\mathbb{R}$ by defining $f:=0$ on $(-\infty, 0)$.

Let $D:=\left\{\left.u \in \mathcal{H}|r \mapsto| r\right|^{\beta} \hat{u}(r) \in L^{2}(\mathbb{R} ; H)\right\}$. Then for $u \in D$ we have

$$
\begin{aligned}
\frac{1}{t}\left(U_{t}^{\beta} u-u\right)^{\wedge}(r) & =\frac{1}{t}\left(\int_{0}^{\infty} U_{s} u \eta_{t}^{\beta}(d s)-u\right)^{\wedge}(r) \\
& =\frac{1}{t}\left(\int_{\mathbb{R}} \mathbb{1}_{[0, \infty)}(\cdot-s) u(\cdot-s) \eta_{t}^{\beta}(d s)-u\right)^{\wedge}(r) \\
& =\frac{1}{t}\left(u * \eta_{t}^{\beta}-u\right)^{\wedge}(r) \\
& =\frac{1}{t} \hat{u}(r)\left(e^{-t(i r)^{\beta}}-1\right) \underset{t \rightarrow 0}{\longrightarrow}-(i r)^{\beta} \hat{u}(r)
\end{aligned}
$$

for $d r$-a.e. $r \in \mathbb{R}$. But since for all $r \in \mathbb{R}$ and $t>0$,

$$
\frac{1}{t}\left|e^{-t(i r)^{\beta}}-1\right| \leq 2|r|^{\beta}
$$

the last convergence also holds in $\mathcal{H}$. Hence $D \subset D(\Lambda, \mathcal{H})$ and

$$
(\Lambda u)^{\wedge}(r)=-(i r)^{\beta} \hat{u}(r), r \in \mathbb{R}
$$

Similarly, one checks that

$$
U_{t}^{\beta} D \subseteq D \quad \forall t>0
$$

and that $(\Lambda, D)$ is closed as an operator from $\mathcal{H}$ to $\mathcal{H}$. Since $D$ is dense in $\mathcal{H}$, (2.10) implies (see [50, Theorem X.49]) that $D$ is an operator core of $(\Lambda, D(\Lambda, \mathcal{H}))$. Consequently, $D=D(\Lambda, \mathcal{H})$ and $\Lambda$ is given by (2.9). Furthermore, we note that for all $\beta>0$

$$
D(\Lambda, \mathcal{H}) \subset H^{\beta}(\mathbb{R} ; H)
$$

by definition of the fractional Sobolev space $H^{\beta}(\mathbb{R} ; H)\left(\subset L^{2}(\mathbb{R} ; H)\right)$, which consists of (Hölder-) continuous functions on $\mathbb{R}$ if $\beta>\frac{1}{2}$. Therefore, if $\beta>\frac{1}{2}$, every $u \in D(\Lambda, \mathcal{H})$ has a continuous version $\tilde{u}$ on $\mathbb{R}$ which is zero on $(-\infty, 0)$, hence $\tilde{u}(0)=0$. 
Next we shall prove a representation formula for $\Lambda$ in terms of the Caputo derivative $\partial_{t}^{\beta}$. To this end, we first define a domain for $\partial_{t}^{\beta}$ which is convenient in our case. Let for $\beta \in(0,1)$

$$
D\left(\partial_{t}^{\beta}\right):=\left\{u \in L^{1}\left([0, \infty) ; V^{*}\right) \mid g_{1-\beta} * u \in W^{1,1}\left([0, T], V^{*}\right) \text { for all } T \in(0, \infty)\right\},
$$

where we define for $u \in L^{1}\left([0, \infty) ; V^{*}\right)$

$$
\left(g_{1-\beta} * u\right)(t):=\frac{1}{\Gamma(1-\beta)} \int_{0}^{t}(t-s)^{-\beta} u(s) d s .
$$

Obviously, for all $T \in(0, \infty)$

$$
g_{1-\beta} * u=\left(\mathbb{1}_{[0, T]} g_{1-\beta}\right) *\left(\mathbb{1}_{[0, \infty)} u\right) \quad \text { on }[0, T],
$$

where for $p \in[1, \infty)$ the latter function belongs to $L^{p}\left(\mathbb{R} ; V^{*}\right)$ if so does $\mathbb{1}_{[0, \infty)} u$, and is in $C\left(\mathbb{R} ; V^{*}\right)$, if in addition $\beta \in\left(0, \frac{p-1}{p}\right)$.

Lemma 2.5. Let $u \in D_{0}:=D(\Lambda, \mathcal{H}) \cap \mathcal{V} \cap D\left(\partial_{t}^{\beta}\right) \cap L^{\infty}([0, \infty) ; H)$. Then

$$
\Lambda u=-\frac{d}{d t}\left(g_{1-\beta} * u\right)
$$

and $D_{0}$ is an operator core for $(\Lambda, D(\Lambda, \mathcal{H}))$.

Proof. First we note that for $T \in[0, \infty)$

$$
\left\|\left(g_{1-\beta} * u\right)(t)\right\|_{V^{*}} \leq \frac{\operatorname{ess} \sup \| u(s)}{\Gamma(1-\beta)(1-\beta)} \|_{V^{*}} t^{1-\beta} \quad \text { for } d t \text {-a.e. } t \in[0, T],
$$

and the same inequality holds with $\|\cdot\|_{H}$ replacing $\|\cdot\|_{V^{*}}$.

From now on we consider all appearing functions, originally only defined on $[0, \infty)$, as functions on all of $\mathbb{R}$ by defining them to be equal to zero on $\mathbb{R} \backslash[0, \infty)$. As in the proof of Proposition 2.4, one can check that $U_{t}^{\beta}\left(D_{0}\right) \subset D_{0}$ (for which, however, it is essential that $\eta_{t}^{\beta}(d s)$ is absolutely continuous with respect to $\left.d s\right)$ and that $D_{0}$ is dense in $\mathcal{H}$. Again applying Theorem X.49 from [50] we obtain that $D_{0}$ is an operator core of $(\Lambda, D(\Lambda, \mathcal{H}))$. Hence it remains to prove (2.12).

Let us start with calculating the Laplace transform $\mathcal{L}$ of the right hand side of (2.12) for any $u \in D\left(\partial_{t}^{\beta}\right) \cap L^{\infty}\left([0, \infty) ; V^{*}\right)$. So let $\lambda \in(0, \infty)$. Then integrating by parts, using (2.13) 
and Fubini's Theorem we obtain

$$
\begin{aligned}
\mathcal{L}\left(\frac{d}{d t}\left(g_{1-\beta} * u\right)\right)(\lambda) & =\int_{0}^{\infty} e^{-\lambda t} \frac{d}{d t}\left(g_{1-\beta} * u\right)(t) d t \\
& =\lim _{T \rightarrow \infty}\left(e^{-\lambda T}\left(g_{1-\beta} * u\right)(T)+\lambda \int_{0}^{T} e^{-\lambda t}\left(g_{1-\beta} * u\right)(t) d t\right) \\
& =\frac{\lambda}{\Gamma(1-\beta)} \int_{0}^{\infty} e^{-\lambda t} \int_{0}^{t}(t-s)^{-\beta} u(s) d s d t \\
& =\frac{\lambda}{\Gamma(1-\beta)} \int_{0}^{\infty} \int_{s}^{\infty}(t-s)^{-\beta} e^{-\lambda t} d t u(s) d s \\
& =\frac{\lambda^{\beta}}{\Gamma(1-\beta)} \int_{0}^{\infty} e^{-\lambda s} u(s) d s \lambda \int_{0}^{\infty}(\lambda t)^{-\beta} e^{-\lambda t} d t \\
& =\lambda^{\beta} \mathcal{L} u(\lambda), \quad \lambda \in(0, \infty) .
\end{aligned}
$$

For the left-hand side of $(2.12)$ and $u \in D_{0}$ we find for all $h \in H, \lambda \in(0, \infty)$, since $\mathcal{L}\left(\eta_{t}^{\beta}\right)(\lambda)=e^{-t \lambda^{\beta}}$

$$
\begin{aligned}
\left\langle\int_{0}^{\infty} \Lambda u(r) e^{-\lambda r} d r, h\right\rangle_{H} & =\lim _{t \rightarrow 0} \frac{1}{t} \int_{0}^{\infty}\left\langle U_{t}^{\beta} u(r)-u(r), h\right\rangle_{H} e^{-\lambda r} d r \\
& =\lim _{t \rightarrow 0} \frac{1}{t}\left(\mathcal{L}\left(\langle u, h\rangle_{H} * \eta_{t}^{\beta}\right)-\mathcal{L}\left(\langle u, h\rangle_{H}\right)\right)(\lambda) \\
& =\lim _{t \rightarrow 0} \frac{1}{t}\left(e^{-t \lambda^{\beta}}-1\right) \mathcal{L}\left(\langle u, h\rangle_{H}\right)(\lambda) \\
& =-\lambda^{\beta}\langle\mathcal{L} u(\lambda), h\rangle_{H} .
\end{aligned}
$$

Hence, $\mathcal{L}(\Lambda(u))(\lambda)=-\lambda^{\beta} \mathcal{L}(u)(\lambda)$ and (2.12) follows.

Proposition 2.6. Let $u \in \mathcal{F}$. Then $u \in D\left(\partial_{t}^{\beta}\right)$ and

$$
\Lambda u=-\frac{d}{d t}\left(g_{1-\beta} * u\right) .
$$

In particular, $g_{1-\beta} * u \in C([0, \infty), H)$.

Proof. By Lemma 2.5 there exist $u_{n} \in D_{0}, n \in \mathbb{N}$, such that as $n \rightarrow \infty$

$$
u_{n} \longrightarrow u \text { in } \mathcal{V}
$$

and

$$
-\partial_{t}^{\beta} u_{n}=\Lambda u_{n} \longrightarrow \Lambda u \text { in } \mathcal{V}^{*}
$$

Let $T \in(0, \infty)$. Obviously, $g_{1-\beta} * u_{n} \longrightarrow g_{1-\beta} * u$ in $L^{\alpha}([0, T] ; V)$ as $n \rightarrow \infty$. Therefore, by completeness $g_{1-\beta} * u \in W^{1, \frac{\alpha}{\alpha-1}}\left([0, T] ; \mathcal{V}^{*}\right)$ and

$$
\Lambda u=-\frac{d}{d t}\left(g_{1-\beta} * u\right) \text { on }[0, T] .
$$

The last part of the assertion then follows by [4, Theorem 1.19, pp.25]. 
Proof of Theorem 2.1: Existence: Let $\varphi$ be as in the assertion of the theorem. Define $\mathcal{A}_{x}$ as $\mathcal{A}$, but with

$$
A_{x}(t, v):=A(t, v+x \varphi(t)), t>0, v \in V
$$

replacing $A$. Then by Theorem 2.3 and Proposition 2.6 there exist $u_{x} \in \mathcal{F}$ such that

$$
\frac{d}{d t}\left(g_{1-\beta} * u_{x}\right)+A_{x} u_{x}=f
$$

Define $u:=u_{x}+x \varphi$. Then $u-x \varphi\left(=u_{x}\right)$ satisfies (2.4) and

$$
\partial_{t}^{\beta}(u-x)+\mathcal{A} u=f \quad d t \text {-a.e. on }(0, T)
$$

and (2.1) is solved. The rest assertions of the Theorem follows from the last part of the assertion of Proposition 2.6 and an elementary fact about convolutions.

Uniqueness: the uniqueness proof is similar to the argument in [62, Theorem 3.1]. So, we only give a brief account of the proof here.

Recall that Riemann-Liouville fractional integral $I_{t}^{\beta} f:=g_{\beta} * f$ and the definition of the Riemann-Liouville kernel

$$
g_{\beta}(t)=\frac{t^{\beta-1}}{\Gamma(\beta)}, t>0 .
$$

Suppose that $u_{1}, u_{2}$ are solutions to (2.1), then $u:=u_{1}-u_{2}$ is a solution to the following equation

$$
\partial_{t}^{\beta} u+A\left(t, u_{1}\right)-A\left(t, u_{2}\right)=0, u(0)=0 .
$$

Let $g^{n} \in W^{1,1}, n \in \mathcal{N}$ be the kernel associated with the Yosida approximation of the operator $\partial_{t}^{\beta}$ (cf. 60, 61]). Then by [60, Theorem 2.1] we have

$$
\begin{aligned}
& \frac{d}{d t}\left(g^{n} *\|u(\cdot)\|_{H}^{2}\right)(t) \leq 2\left\langle\frac{d}{d t}\left(g^{n} * u\right)(t), u(t)\right\rangle_{H} \\
= & 2_{V^{*}}\left\langle\frac{d}{d t}\left(g_{1-\beta} * u\right)(t), u(t)\right\rangle_{V}+2_{V^{*}}\left\langle\frac{d}{d t}\left(g^{n} * u\right)(t)-\frac{d}{d t}\left(g_{1-\beta} * u\right)(t), u(t)\right\rangle_{V} \\
= & 2_{V^{*}}\left\langle A\left(t, u_{1}(t)\right)-A\left(t, u_{2}(t)\right), u(t)\right\rangle_{V}+2_{V^{*}}\left\langle\frac{d}{d t}\left(g^{n} * u\right)(t)-\frac{d}{d t}\left(g_{1-\beta} * u\right)(t), u(t)\right\rangle_{V} \\
\leq & 2_{V^{*}}\left\langle\frac{d}{d t}\left(g^{n} * u\right)(t)-\frac{d}{d t}\left(g_{1-\beta} * u\right)(t), u(t)\right\rangle_{V}=: 2 h^{n}(t) .
\end{aligned}
$$

Note that $h^{n} \rightarrow 0$ in $L^{1}([0, T])$, which yields that $g_{\beta} * h^{n} \rightarrow 0$ in $L^{1}([0, T])$ (cf. [60]). Moreover, we have

$$
g_{\beta} * \frac{d}{d t}\left(g^{n} *\|u(\cdot)\|_{H}^{2}\right)=\frac{d}{d t}\left(g^{n} * g_{\beta} *\|u(\cdot)\|_{H}^{2}\right) \rightarrow \frac{d}{d t}\left(g_{1-\beta} * g_{\beta} *\|u(\cdot)\|_{H}^{2}\right)=\|u(\cdot)\|_{H}^{2}
$$

in $L^{1}([0, T])$ as $n \rightarrow \infty$.

Hence, by applying convolution with the kernel $g_{\beta}$ to (2.14) we have

$$
\|u(t)\|_{H}^{2} \leq 0 \text {, a.e.t } \in[0, T],
$$


which implies that $u_{1}=u_{2}$, i.e. the solution to (2.1) is unique.

Suppose that $U$ is a Hilbert space and $W(t)$ is a $U$-valued cylindrical Wiener process defined on a filtered probability space $\left(\Omega, \mathcal{F}, \mathcal{F}_{t}, \mathbb{P}\right)$. Now we consider stochastic nonlinear evolution equations with fractional time derivative of type

$$
\partial_{t}^{\beta}(X(t)-x)+A(t, X(t))=\partial_{t}^{\gamma} \int_{0}^{t} B(s) d W(s), 0<t<T,
$$

where $\gamma \in(0,1], B:[0, T] \rightarrow L_{H S}(U ; H)$ is measurable (here $\left(L_{H S}(U ; H),\|\cdot\|_{H S}\right)$ denotes the space of all Hilbert-Schmidt operators from $U$ to $H$ ).

We want to explain the reason why this form of random noise term is used in (2.15). In fact, heuristically

$$
\partial_{t}^{\gamma} \int_{0}^{t} B(s) d W(s)=I_{t}^{1-\gamma}[B(t) d W(t)]
$$

which can be used to model systems subject to classical random noise (the case $\gamma=1$ ) or random noise with certain memory effects (the case $\gamma<1$ ). This form of noise will naturally appear if e.g. you consider the heat transfer with random effects with memory (see [18]) or use the time fractional Duhamel's principle to derive the appropriate form of stochastic time-fractional diffusion equations (see [43] for the case $\gamma=\beta$ ). The case $\gamma=\beta$ is investigated in various papers about stochastic heat type or wave type equations, see e.g. [15, 17, 24, 43, 44], and the case $\gamma=1$ is studied in [16, 26].

In this work we consider the general case $\gamma<\beta+\frac{1}{2}$, this assumption is natural since the stochastic integral term in (2.16) is well-defined if and only if $(e . g$. assuming $B(\cdot)$ is bounded)

$$
\int_{0}^{t}(t-s)^{2(\beta-\gamma)}\|B(s)\|_{H S}^{2} d s \leq C \int_{0}^{t}(t-s)^{2(\beta-\gamma)} d s<\infty,
$$

which is equivalent to $\gamma<\beta+\frac{1}{2}$. The same assumption $\gamma<\beta+\frac{1}{2}$ is also used in [18] which is deduced there by a differentiability argument.

For a concise formulation of our result we define for $t \in[0, \infty)$

$$
F(t):=\frac{1}{\Gamma(1+\beta-\gamma)} \int_{0}^{t}(t-s)^{\beta-\gamma} B(s) d W(s) .
$$

Theorem 2.7. Suppose that $\gamma \in(0,1], T \in[0, \infty)$ and that $A$ satisfies $(H 1)-(H 4), B \in$ $L^{\infty}\left([0, T], L_{H S}(U ; H)\right)$ if $\gamma<\beta+\frac{1}{2}$ or $B \in L^{2}\left([0, T], L_{H S}(U ; H)\right)$ if $\gamma \leq \beta$. Assume also that $F \in V, d t \otimes \mathbb{P}$-a.e. (which is e.g. the case if $B(t)$ is a Radonifying map from $U$ to $V$ ). Then for every $x \in V$ (2.15) has a unique $\mathcal{F}_{t}$-adapted solution $X$ such that $X-F-x \varphi \in \mathcal{F}$, $\mathbb{P}$-a.s. for every $\varphi \in L^{\alpha}([0, \infty) ; \mathbb{R})$ with $\varphi \equiv 1$ on $[0, T+1)$. In particular,

$$
X-F-x \varphi \in L^{\alpha}([0, \infty) ; V) ; \partial_{t}^{\beta}(X-F-x \varphi) \in L^{\frac{\alpha}{\alpha-1}}\left([0, \infty) ; V^{*}\right), \mathbb{P} \text {-a.s }
$$

and for dt-a.e. $t \in[0, T]$,

$X(t)=x-\frac{1}{\Gamma(\beta)} \int_{0}^{t}(t-s)^{\beta-1} A(s, X(s)) d s+\frac{1}{\Gamma(1+\beta-\gamma)} \int_{0}^{t}(t-s)^{\beta-\gamma} B(s) d W(s), \mathbb{P}-a . s$. 
Furthermore, $t \mapsto \frac{1}{\Gamma(1-\beta)} \int_{0}^{t}(t-s)^{-\beta}(X(s)-x \varphi(s)) d s$ and $F$ have continuous $H$-valued $d t$-versions, and $t \mapsto X(t)$ has a continuous $V^{*}$-valued $d t$-version if $\beta \in\left(\frac{\alpha-1}{\alpha}, 1\right)$.

Proof. Let $u(t)=X(t)-F(t)$, then $u(t)$ satisfies the following equation

$$
\partial_{t}^{\beta}(u(t)-x)+A(t, u(t)+F(t))=0,0<t<T .
$$

Define

$$
\tilde{A}(t, u)=A(t, u+F(t)), u \in V .
$$

Since $F \in V d t \otimes \mathbb{P}$-a.e., it is easy to see that $\tilde{A}$ still satisfies (H1)-(H4). Hence (2.17) has a unique solution $u$, which implies that $u+F$ is the unique solution to (2.15).

The $\mathcal{F}_{t}$-adaptiveness of the solution follows by the proofs of Theorem 2.3 and Lemma 2.2. Since $g_{\alpha} * g_{\beta}=g_{\alpha+\beta}$, it easily follows (e.g. by [35, Proposition 6.3.3]) that $F$ is $\mathbb{P}$-a.s. continuous in $H$. Hence $X$ is continuous in $V^{*}$ if $\beta \in\left(\frac{\alpha-1}{\alpha}, 1\right)$.

Remark 2.3. From the above proof one can see that Theorem 2.7 also holds for the case that $A$ and $B$ are random coefficients (i.e. also depend on $\omega \in \Omega$ ) in a progressively measurable way (cf. [35, Section 4.1]). It's also obvious that one could take the initial condition $x$ as some $V$-valued random variable.

\section{Application to Examples}

In this part we will apply our main result to establish the existence and uniqueness of solutions to (stochastic) porous medium equations and $p$-Laplace equations with time-fractional derivative. Both are open problems even in the deterministic case. In the recent work [61, the authors derive very nice decay estimates for the solution of those equations (by assuming the existence) and the decay behaviour turns out to be notably different from the classical parabolic case. As said, here we give a positive answer to the question concerning the existence and uniqueness of solutions to the time-fractional porous medium equations and $p$-Laplace equations.

Both examples are just model cases which we present here motivated by [61]. There are many more examples (see e.g. [5] and [51]). In particular, fast diffusion equations are also covered. We are confident that we can extend our approach to the case of multi-valued operators as well.

Let $\Lambda \subset \mathbb{R}^{d}$ be an open bounded domain and $\Delta$ be the Laplace operator, and for $p \in[1, \infty[$ we use $L^{p}(\Lambda)$ and $H_{0}^{n, p}(\Lambda)$ to denote the $p$ th-integrable Lebesgue space and the Sobolev space of order $n$ in $L^{p}(\Lambda)$ with Dirichlet boundary conditions. Recall that $X^{*}$ denotes the dual space of a Banach space $X$.

\subsection{Time-fractional porous medium equation}

We first introduce the porous medium operator $A(u):=\Delta \Psi(u)$. Let $\Psi: \mathbb{R} \rightarrow \mathbb{R}$ be a function having the following properties: 
( $\Psi 1) \Psi$ is continuous.

( $\Psi 2)$ For all $s, t \in \mathbb{R}$

$$
(t-s)(\Psi(t)-\Psi(s)) \geq 0 .
$$

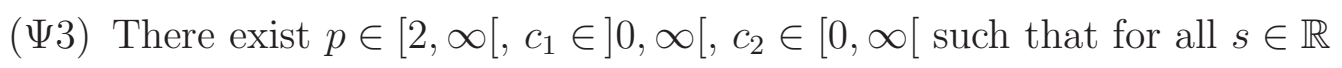

$$
s \Psi(s) \geq c_{1}|s|^{p}-c_{2} .
$$

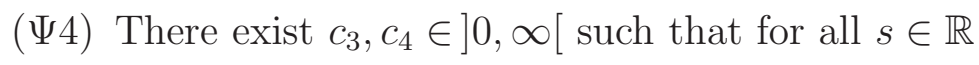

$$
|\Psi(s)| \leq c_{3}|s|^{p-1}+c_{4},
$$

where $p$ is as in $(\Psi 3)$.

Now we consider the following Gelfand triple

$$
V:=L^{p}(\Lambda) \subseteq H:=\left(H_{0}^{1,2}(\Lambda)\right)^{*} \subseteq V^{*}:=\left(L^{p}(\Lambda)\right)^{*}
$$

and stochastic time-fractional porous medium equation

$$
\partial_{t}^{\beta}(X(t)-x)=\Delta \Psi(X(t))+\partial_{t}^{\gamma} \int_{0}^{t} B(s) \mathrm{d} W(s), 0<t<T .
$$

Remark 3.1. Since here $H$ is not taken as $L^{2}(\Lambda)$, by the definition of the Gelfand triple, one should note that

$$
V^{*} \neq L^{\frac{p}{p-1}}(\Lambda) .
$$

In fact, one can prove that (see [35, Lemma 4.1.13])

$$
V^{*}=\Delta\left(L^{\frac{p}{p-1}}(\Lambda)\right)
$$

Theorem 3.1. Suppose that $\Psi$ satisfies $(\Psi 1)-(\Psi 4)$. Then Theorem 2.7 applies with $A:=$ $\Delta \Psi(\cdot)$ and $\alpha:=p$.

Proof. Now we can define the porous medium operator $A: V \rightarrow V^{*}$ by

$$
A(u):=\Delta \Psi(u), \quad u \in L^{p}(\Lambda) .
$$

Note that by [35, Lemma 4.1.13] the operator $A$ is well-defined. The conclusion follows directly from Theorem 2.7 by checking $(\mathrm{H} 1)-(\mathrm{H} 4)$ hold for $-A$.

In fact, it is well-known that the porous medium operator satisfies the monotonicity and coercivity properties (see e.g. [35, Section 4.1]). We include the proof here for the reader's convenience. 
(H1): Let $u, v, x \in V=L^{p}(\Lambda)$ and $\lambda \in \mathbb{R}$. Then by

$$
\begin{aligned}
V^{*}\langle A(u+\lambda v), x\rangle_{V} & ={ }_{V^{*}}\langle\Delta \Psi(u+\lambda v), x\rangle_{V} \\
& =-\int_{\Lambda} \Psi(u(\xi)+\lambda v(\xi)) x(\xi) \mathrm{d} \xi .
\end{aligned}
$$

By $(\Psi 4)$ for $|\lambda| \leq 1$ the integrand in the right-hand side of (3.3) is bounded by

$$
\left[c_{4}+c_{3} 2^{p-2}\left(|u|^{p-1}+|v|^{p-1}\right)\right]|x|
$$

which by Hölder's inequality is in $L^{1}(\Lambda)$. So, (H1) follows by $(\Psi 1)$ and Lebesgue's dominated convergence theorem.

(H2): Let $u, v \in V=L^{p}(\Lambda)$. Then

$$
\begin{aligned}
\left.V^{*}\langle A(u)-A(v), u-v)\right\rangle_{V} & ={ }_{V^{*}}\langle\Delta(\Psi(u)-\Psi(v)), u-v\rangle_{V} \\
& =-\int_{\Lambda}[\Psi(u(\xi))-\Psi(v(\xi))](u(\xi)-v(\xi)) \mathrm{d} \xi \\
& \leq 0
\end{aligned}
$$

where we used ( $\Psi 2)$ in the last step.

(H3): Let $v \in L^{p}(\Lambda)=V$. Then by $(\Psi 3)$

$$
\begin{aligned}
V^{*}\langle A(v), v\rangle_{V} & =-\int_{\Lambda} \Psi(v(\xi)) v(\xi) \mathrm{d} \xi \\
& \leq \int_{\Lambda}\left(-c_{1}|v(\xi)|^{p}+c_{2}\right) \mathrm{d} \xi
\end{aligned}
$$

Hence (H3) is satisfied with $\delta:=c_{1}, \alpha=p$ and $g(t)=c_{2}|\Lambda|$.

(H4): Let $v \in L^{p}(\Lambda)=V$. Then by [35, Lemma 4.1.13] and $(\Psi 4)$

$$
\begin{aligned}
\|A(v)\|_{V^{*}} & =\|\Delta \Psi(v)\|_{V^{*}} \\
& =\|\Psi(v)\|_{L^{\frac{p}{p-1}}} \\
& \leq c_{4}|\Lambda|^{\frac{p-1}{p}}+c_{3}\left(\int|v(\xi)|^{p} \mathrm{~d} \xi\right)^{\frac{p-1}{p}} \\
& =c_{4}|\Lambda|^{\frac{p-1}{p}}+c_{3}\|v\|_{V}^{p-1}
\end{aligned}
$$

so (H4) holds with $\alpha=p$. 
Remark 3.2. (i) For $p \in\left[2, \infty\left[\right.\right.$ and $\Psi(s):=s|s|^{p-2}$ we have

$$
A(v)=\Delta\left(v|v|^{p-2}\right), v \in L^{p}(\Lambda),
$$

which is the non-linear operator appearing in the classical porous medium equation (cf. e.g. [58, 59]), i.e.

$$
\frac{\partial u(t)}{\partial t}=\Delta\left(u(t)|u(t)|^{p-2}\right), \quad u(0, \cdot)=u_{0},
$$

whose solution describes the time evolution of the density $u(t)$ of a substance in a porous medium. And as a matter of fact, the time-fractional porous medium equation was first introduced by Caputo in [13] to describe the diffusion of fluids in porous media with memory.

(ii) As mentioned before, our results apply to the situation in [51] where $\Delta \Psi(u)$ is replaced by $L \Psi(u)$, here $L$ is the generator of a transient Dirichlet form on $L^{2}(E, \mathcal{E}, \mu)$ for abstract $\sigma$-finite measure spaces $(E, \mathcal{E}, \mu)$, so (2.15) takes the form

$$
\partial_{t}^{\beta}(X(t)-x)-L \Psi(X(t))=\partial_{t}^{\gamma} \int_{0}^{t} B(s) \mathrm{d} W(s) .
$$

This, in particular, includes "fractal" (i.e. non-local) operators $L$, as e.g. fractional Laplacian $-(-\Delta)^{\alpha}, \alpha \in(0,1] \cap\left(0, \frac{d}{2}\right)$, and the underlying domain $\Lambda$ may be unbounded, e.g. $\Lambda=\mathbb{R}^{d}$, $d \geq 3$. $\Psi$ may belong to the more general class described in [51], but must be monotone. In particular, $\Psi(r):=r|r|^{m-1}$ with $m \in(0,1]$ is covered, i.e. the fast diffusion equation. So, equations as

$$
\partial_{t}^{\beta}(X(t)-x)+(-\Delta)^{\alpha}\left(X(t)|X(t)|^{m-1}\right)=\partial_{t}^{\gamma} \int_{0}^{t} B(s) \mathrm{d} W(s)
$$

for $m \in(0, \infty)$ are covered.

\subsection{Time-fractional $p$-Laplace equation}

Now we consider stochastic time-fractional $p$-Laplace equations $(p \geq 2)$

$$
\partial_{t}^{\beta}(X(t)-x)=\operatorname{div}\left(|\nabla X(t)|^{p-2} \nabla X(t)\right)+\partial_{t}^{\gamma} \int_{0}^{t} B(s) \mathrm{d} W(s), 0<t<T .
$$

We will choose the follwing Gelfand triple

$$
V:=H_{0}^{1, p}(\Lambda) \subseteq H:=L^{2}(\Lambda) \subseteq V^{*}=\left(H_{0}^{1, p}(\Lambda)\right)^{*}
$$

and define $A: H_{0}^{1, p}(\Lambda) \rightarrow H_{0}^{1, p}(\Lambda)^{*}$ by

$$
A(u):=\operatorname{div}\left(|\nabla u|^{p-2} \nabla u\right), u \in H_{0}^{1, p}(\Lambda) ;
$$

more precisely, given $u \in H_{0}^{1, p}(\Lambda)$, then we define

$$
V^{*}\langle A(u), v\rangle_{V}:=-\int_{\Lambda}|\nabla u(\xi)|^{p-2}\langle\nabla u(\xi), \nabla v(\xi)\rangle \mathrm{d} \xi \quad \text { for all } v \in H_{0}^{1, p}(\Lambda) .
$$


Here $A$ is called the $p$-Laplacian, also denoted by $\Delta_{p}$. Note that $\Delta_{2}=\Delta$.

It is well-known that $A: V \rightarrow V^{*}$ is well-defined (see e.g. [35, Section 4.1]). In fact, we only need to show that the right-hand side of (3.6) defines a linear functional in $v \in V$ which is continuous with respect to \|\|$_{V}=\|\|_{1, p}$. First we recall that $\nabla u \in L^{p}\left(\Lambda ; \mathbb{R}^{d}\right)$ for all $u \in H_{0}^{1, p}(\Lambda)$. Hence by Hölder's inequality

$$
\begin{aligned}
\int|\nabla u(\xi)|^{p-1}|\nabla v(\xi)| \mathrm{d} \xi & \leq\left(\int|\nabla u(\xi)|^{p} \mathrm{~d} \xi\right)^{\frac{p-1}{p}}\left(\int|\nabla v(\xi)|^{p} \mathrm{~d} \xi\right)^{\frac{1}{p}} \\
& \leq\|u\|_{1, p}^{p-1}\|v\|_{1, p} .
\end{aligned}
$$

Since this dominates the absolute value of the right-hand side of (3.6) for all $u \in H_{0}^{1, p}(\Lambda)$, we have that $A(u)$ is a well-defined element of $\left(H_{0}^{1, p}(\Lambda)\right)^{*}$ and that

$$
\|A(u)\|_{V^{*}} \leq\|u\|_{V}^{p-1} .
$$

Theorem 3.2. Suppose that $p \geq 2$. Then Theorem 2.7 applies with $A$ as defined in (3.5) and $\alpha:=p$.

Proof. The conclusion follows directly from Theorem 2.7 by checking (H1)-(H4) hold for $-A$ here. Now we include the proof here also for the reader's convenience.

(H1): Let $u, v, x \in H_{0}^{1, p}(\Lambda)$, then by (3.6) we have to show for $\lambda \in \mathbb{R},|\lambda| \leq 1$

$$
\begin{aligned}
& \lim _{\lambda \rightarrow 0} \int_{\Lambda}\left(|\nabla(u+\lambda v)(\xi)|^{p-2}\langle\nabla(u+\lambda v)(\xi), \nabla x(\xi)\rangle\right. \\
& \left.\quad-|\nabla u(\xi)|^{p-2}\langle\nabla u(\xi), \nabla x(\xi)\rangle\right) \mathrm{d} \xi=0 .
\end{aligned}
$$

Since obviously the integrands converge to zero as $\lambda \rightarrow 0 \mathrm{~d} \xi$-a.e., we only have to find a dominating function to apply Lebesgue's dominated convergence theorem. But obviously, since $|\lambda| \leq 1$

$$
\begin{aligned}
& |\nabla(u+\lambda v)(\xi)|^{p-2}|\langle\nabla(u+\lambda v)(\xi), \nabla x(\xi)\rangle| \\
\leq & 2^{p-2}\left(|\nabla u(\xi)|^{p-1}+|\nabla v(\xi)|^{p-1}\right)|\nabla x(\xi)|
\end{aligned}
$$

and the right-hand side is in $L^{1}(\Lambda)$ by Hölder's inequality as we have seen above. 
(H2): Let $u, v \in H_{0}^{1, p}(\Lambda)$. Then by (3.6)

$$
\begin{aligned}
& -V^{*}\langle A(u)-A(v), u-v\rangle_{V} \\
= & \int_{\Lambda}\left\langle|\nabla u(\xi)|^{p-2} \nabla u(\xi)-|\nabla v(\xi)|^{p-2} \nabla v(\xi), \nabla u(\xi)-\nabla v(\xi)\right|>\mathrm{d} \xi \\
= & \int_{\Lambda}\left(|\nabla u(\xi)|^{p}+|\nabla v(\xi)|^{p}-|\nabla u(\xi)|^{p-2}\langle\nabla u(\xi), \nabla v(\xi)\rangle\right. \\
& \left.\quad-|\nabla v(\xi)|^{p-2}\langle\nabla u(\xi), \nabla v(\xi)\rangle\right) \mathrm{d} \xi \\
\geq & \int_{\Lambda}\left(|\nabla u(\xi)|^{p}+|\nabla v(\xi)|^{p}-|\nabla u(\xi)|^{p-1}|\nabla v(\xi)|\right. \\
& \left.\quad-|\nabla v(\xi)|^{p-1}|\nabla u(\xi)|\right) \mathrm{d} \xi \\
= & \int_{\Lambda}\left(|\nabla u(\xi)|^{p-1}-|\nabla v(\xi)|^{p-1}\right)(|\nabla u(\xi)|-|\nabla v(\xi)|) \mathrm{d} \xi \\
\geq & 0
\end{aligned}
$$

since the map $\mathbb{R}_{+} \ni s \mapsto s^{p-1}$ is increasing. Hence (H2) is proved.

(H3): Because $\Lambda$ is bounded by Poincaré's inequality there exists a constant $c=c(p, d,|\Lambda|) \in$ ] $0, \infty[$ such that

$$
\int_{\Lambda}|\nabla u(\xi)|^{p} \mathrm{~d} \xi \geq c \int_{\Lambda}|u(\xi)|^{p} \mathrm{~d} \xi \quad \text { for all } u \in H_{0}^{1, p}(\Lambda) .
$$

Hence by (3.6) for all $u \in H_{0}^{1, p}(\Lambda)$

$$
V^{*}\langle A(u), u\rangle_{V}=-\int_{\Lambda}|\nabla u(\xi)|^{p} \mathrm{~d} \xi \leq-\frac{\min (1, c)}{2}\|u\|_{1, p}^{p}
$$

So, (H3) holds with $\alpha=p$.

(H4): This condition holds for $A$ by (3.7) with $\alpha=p$.

Remark 3.3. $(i)$ It is easy to show that the above result also holds for the more general case where $\Delta_{p}$ is replaced by

$$
A(u):=\operatorname{div}(\Psi(|\nabla X(t)|) \nabla X(t)),
$$

where $\Psi$ satisfies $(\Psi 1)-(\Psi 4)$ with some $p>1$. Moreover, one can further generalize to more general quasilinear differential operator

$$
A(u):=\sum_{|i| \leq m}(-1)^{|i|} D_{i} A_{i}(t, x, D u(t, x)), \text { where } D u=\left(D_{j} u\right)_{|j| \leq m} .
$$

Under certain assumptions the above operator also satisfies (H1)-(H4) (cf. e.g. 64, Proposition 30.10]). 
(ii) In the case of the $p$-Laplacian or more general operators, it is possible to add some monomials up to order $p$ as pertubation. For example, since $H_{0}^{1, p}(\Lambda) \subset L^{p}(\Lambda)$ is continuous and dense, so

$$
A(u):=\operatorname{div}\left(|\nabla u|^{p-2} \nabla u\right)-u|u|^{p-2}, u \in H_{0}^{1, p}(\Lambda),
$$

still satisfies (H1)-(H4) with respect to the Gelfand triple

$$
H_{0}^{1, p}(\Lambda) \subset L^{2}(\Lambda) \subset\left(H_{0}^{1, p}(\Lambda)\right)^{*}
$$

\section{Acknowledgements}

The second named author would like to thank his hosts at Madeira University for a very pleasant stay in summer 2017 where part of this work was done. Some helpful comments and suggestions from the referee are also gratefully acknowledged.

\section{References}

[1] M. Allen, L. Caffarelli, A. Vasseur, A parabolic problem with a fractional time derivative, Arch. Ration. Mech. Anal. 221 (2016), no. 2, 603-630.

[2] B. Baeumer, M.M. Meerschaert, E. Nane, Brownian subordinators and fractional Cauchy problems, Trans. Amer. Math. Soc. 361 (2009), 3915-3930.

[3] D. Baleanu, K. Diethelm, E. Scalas, J.J. Trujillo, Fratcional Calculus: Models and Numerical Methods, World Scientific, Singapore, 2012.

[4] V. Barbu, Nonlinear differential equations of monotone types in Banach spaces, Springer Monographs in Mathematics, Springer, New York, 2010.

[5] V. Barbu, G. Da Prato, M. Röckner, Stochastic Porous Media Equations, monograph, Lecture Notes in Mathematics 2163, Springer, 2016.

[6] V. Barbu, M. Röckner, An operatorial approach to stochastic partial differential equations driven by linear multiplicative noise. J. Eur. Math. Soc. 17 (2015), 1789-1815.

[7] A. Bensoussan and R. Temam, Equations stochastiques de type Navier-Stokes, J. Funct. Anal. 13 (1973), 195-222.

[8] H. Brézis, Équations et inéquations non linéaires dans les espaces vectoriels en dualité, Ann. Inst. Fourier 18 (1968), 115-175.

[9] H. Brézis, Opérateurs maximaux monotones, North-Holland, Amsterdam, 1973.

[10] F. E. Browder, Non-linear equations of evolution, Ann. Math. 80 (1964), 485-523. 
[11] F. E. Browder, Pseudo-monotone operators and nonlinear elliptic boundary value problems on unbounded domains, Proc. Natl. Acad. Sci. USA 74 (1977), 2659-2661.

[12] Z. Brzeźniak, W. Liu and J. Zhu, Strong solutions for SPDE with locally monotone coefficients driven by Lévy noise, Nonlinear Anal. Real World Appl. 17 (2014), 283310.

[13] M. Caputo, Diffusion of fluids in porous media with memory. Geothermics 28 (1999), $113-130$.

[14] M. Caputo, Linear models of dissipation whose $Q$ is almost frequency independent-II. Geophys. J. R. Astr. Soc. 13 (1967), 529-539.

[15] L. Chen, Nonlinear stochastic time-fractional diffusion equations on $\mathbb{R}$ : moments, Hölder regularity and intermittency. Trans. Amer. Math. Soc. 369 (2017), no. 12, 84978535.

[16] L. Chen, G. Hu, Y. Hu, J. Huang, Space-time fractional diffusions in Gaussian noisy environment. Stochastics 89 (2017), no. 1, 171-206.

[17] L. Chen, Y. Hu, D. Nualart. Nonlinear stochastic time-fractional slow and fast diffusion equations. Preprint, 2015.

[18] Z.-Q. Chen, K.-H. Kim, P. Kim, Fractional time stochastic partial differential equations. Stoch. Process. Appl. 125 (2015), 1470-1499.

[19] D. Craiem, F. J. Rojo, J. M. Atienza, R. L. Armentano, and G. V. Guinea. Fractional order viscoelasticity applied to describe uniaxial stress relaxation of human arteries. Phys. Med. Biol., 53 (2008), 4543.

[20] J. Cui, L. Yan, Existence result for fractional neutral stochastic integro-differential equations with infinite delay, Journal of Physics A: Mathematical and Theoretical, 44 (2011), no. 33, 335201, 16pp.

[21] K. Diethelm. The analysis of fractional differential equations. An application-oriented exposition using differential operators of Caputo type. Lecture Notes in Mathematics, Springer-Verlag, Berlin, 2010.

[22] M. Doi and S. Edwards. The Theory of Polymer Dynamics. International series of monographs on physics. Clarendon Press, 1988.

[23] J. Ferry. Viscoelastic Properties of Polymers. Wiley, 1980.

[24] M. Foondun, E. Nane. Asymptotic properties of some space-time fractional stochastic equations. Math. Z. 287 (2017), no. 1-2, 493-519.

[25] I. Gyöngy, On stochastic equations with respect to semimartingale III, Stochastics 7 (1982), 231-254. 
[26] G. Hu, Y. Hu, Fractional diffusion in Gaussian noisy environment. Preprint arXiv:1502.05514v1, 2015.

[27] R. Herrmann, Fractional Calculus: An Introduction for Physicists, World Scientific, Singapore, 2011.

[28] V.G. Jakubowski, P. Wittbold, On a nonlinear elliptic-parabolic integro-differential equation with $L^{1}$-data, J. Differential Equations 197 (2004), 427-445.

[29] N.V. Krylov and B. L. Rozovskii, Stochastic evolution equations, Translated from Itogi Naukii Tekhniki, Seriya Sovremennye Problemy Matematiki 14 (1979), 71-146.

[30] J.-L. Lions, Quelques méthodes de résolution des problèmes aux limites non linéaires, Dunod, Paris, 1969.

[31] W. Liu, Existence and uniqueness of solutions to nonlinear evolution equations with locally monotone operators, Nonlinear Anal. 74 (2011), 7543-7561.

[32] W. Liu, Well-posedness of stochastic partial differential equations with Lyapunov conditions, J. Differential Equations 255 (2013), 572-592

[33] W. Liu and M. Röckner, SPDE in Hilbert space with locally monotone coefficients., J. Funct. Anal. 259 (2010), 2902-2922.

[34] W. Liu and M. Röckner, Local and global well-posedness of SPDE with generalized coercivity conditions, J. Differential Equations 254 (2013), 725-755.

[35] W. Liu and M. Röckner, Stochastic Partial Differential Equations: An Introduction, Universitext, Springer, 2015.

[36] R. L. Magin. Fractional calculus models of complex dynamics in biological tissues. Comput. Math. Appl., 59 (2010), 1586-1593.

[37] F. Mainardi. Fractional calculus and waves in linear viscoelasticity. Imperial College Press, London, 2010.

[38] Z. Ma, M. Röckner, Introduction to the theory of (nonsymmetric) Dirichlet forms, Universitext. Springer-Verlag, Berlin, 1992.

[39] M.M. Meerschaert, E. Nane, P. Vellaisamy, Fractional Cauchy problems on bounded domains, Ann. Probab. 37 (2009), 979-1007.

[40] M.M. Meerschaert, A. Sikorskii, Stochastic Models for Fractional Calculus, in: De Gruyter Studies in Mathematics, vol. 43, Walter de Gruyter, Berlin/Boston, 2012.

[41] R. Metzler, J. Klafter, The restaurant at the end of the random walk: recent developments in the description of anomalous transport by fractional dynamics, J. Phys. A 37 (2004), 161-208. 
[42] R. Metzler, J. Klafter, The random walks guide to anomalous diffusion: a fractional dynamics approach. Phys. Rep. 339 (2000), 1-77.

[43] J. Mijena, E. Nane, Space-time fractional stochastic partial differential equations. Stoch. Process Appl. 125 (2015), 3301-3326.

[44] J. Mijena, E. Nane, Intermittence and space-time fractional stochastic partial differential equations. Potential Anal. 44 (2016), 295-312.

[45] A. Mura, Non-Markovian Stochastic Processes and their Applications: from Anomalous Diffusion to Time Series Analysis. PhD thesis. Università di Bologna. 2008.

[46] E. Orsingher, L. Beghin, Fractional diffusion equations and processes with randomly varying time, Ann. Probab. 37 (2009), 206-249.

[47] E. Pardoux, Equations aux dérivées partielles stochastiques non linéaires monotones, Ph.D. thesis, Université Paris XI, 1975.

[48] I. Podlubny. Fractional differential equations, volume 198 of Mathematics in Science and Engineering. Academic Press Inc., San Diego, CA, 1999.

[49] J. Prüss, Evolutionary Integral Equations and Applications. Monographs in Mathematics, vol. 87. Birkhäser, Basel, 1993.

[50] M. Reed, B. Simon, Methods of modern mathematical physics. II. Fourier analysis, self-adjointness. Academic Press, New York-London, 1975.

[51] J. Ren, M. Röckner, and F.-Y. Wang, Stochastic generalized porous media and fast diffusion equations, J. Differential Equations 238 (2007), no. 1, 118-152.

[52] B. Ross, The development of fractional calculus 1695-1900. Hist. Math. 4 (1977), 75-89.

[53] S.G. Samko, A.A. Kilbas, O.I. Marichev, Fractional Integrals and Derivatives, Gordon and Breach, 1993.

[54] M. Shlesinger, J. Klafter, Y.M. Wong, Random walks with infinite spatial and temporal moments, J. Statist. Phys. 27 (1982), 499-512.

[55] R.E. Showalter, Monotone operators in Banach space and nonlinear partial differential equations, Mathematical Surveys and Monographs, vol. 49, American Mathematical Society, Providence, 1997.

[56] R. Sakthivel, P. Revathi, Y. Ren, Existence of solutions for nonlinear fractional stochastic differential equations, Nonlinear Analysis: Theory, Methods \& Applications. 81(2013), 70-86.

[57] W. Stannat, The theory of generalized Dirichlet forms and its applications in analysis and stochastics. Mem. Amer. Math. Soc. 142 (1999), no. 678, 101 pp. 
[58] J.L. Vázquez, Smoothing and decay estimates for nonlinear diffusion equations. Equations of porous medium type. Oxford Lecture Series in Mathematics and its Applications, 33. Oxford University Press, Oxford, 2006.

[59] J.L. Vázquez, The porous medium equation. Mathematical theory. Oxford Mathematical Monographs. Oxford University Press, Oxford, 2007.

[60] V. Vergara, R. Zacher, Lyapunov functions and convergence to steady state for differential equations of fractional order, Math. Z. 259 (2008), 287-309.

[61] V. Vergara, R. Zacher, Optimal decay estimates for time-fractional and other nonlocal subdiffusion equations via energy methods, SIAM J. Math. Anal. 47 (2015), no. 1, 210239.

[62] R. Zacher, Weak solutions of abstract evolutionary integro-differential equations in Hilbert spaces, Funkcialaj Ekvacioj 52 (2009), 1-18.

[63] R. Zacher, Global strong solvability of a quasilinear subdiffusion problem, J. Evol. Equ. 12 (2012), 813-831.

[64] E. Zeidler, Nonlinear functional analysis and its applications, II/B, nonlinear monotone operators, Springer-Verlag, New York, 1990.

[65] X. Zhang, On stochastic evolution equations with non-Lipschitz coefficients, Stoch. Dyn. 9 (2009), no. 4, 549-595.

[66] X. Zhang, Stochastic Volterra equations in Banach spaces and stochastic partial differential equation, J. Funct. Anal. 258 (2010), no. 4, 1361-1425.

[67] Y. Zhou, Basic theory of fractional differential equations. World Scientific Publishing, Hackensack, NJ, 2014. 